\title{
Equalities between greatest common divisors involving three coprime pairs
}

\author{
Rogelio Tomás García \\ CERN \\ Geneva, Switzerland \\ e-mail: rogelio.tomas@cern.ch
}

\begin{abstract}
A new equality of the greatest common divisor ( $\mathrm{gcd}$ ) of quantities involving three coprime pairs is proven in this note. For $a_{i}$ and $b_{i}$ positive integers such that $\operatorname{gcd}\left(a_{i}, b_{i}\right)=1$ for $i \in\{1,2,3\}$ and $d_{i j}=\left|a_{i} b_{j}-a_{j} b_{i}\right|$, then $\operatorname{gcd}\left(d_{32}, d_{31}\right)=\operatorname{gcd}\left(d_{32}, d_{21}\right)=\operatorname{gcd}\left(d_{31}, d_{21}\right)$. The proof uses properties of Farey sequences.
\end{abstract}

Keywords: Greatest common divisor, Farey, Equality.

2010 Mathematics Subject Classification: 11A05, 11 B57.

\section{Definitions}

The Farey sequence $F_{N}$ of order $N$ is an ascending sequence of irreducible fractions between 0 and 1 whose denominators do not exceed $N$ [1]. For example, $F_{5}$ is as follows,

$$
F_{5}=\left\{\frac{0}{1}, \frac{1}{5}, \frac{1}{4}, \frac{1}{3}, \frac{2}{5}, \frac{1}{2}, \frac{3}{5}, \frac{2}{3}, \frac{3}{4}, \frac{4}{5}, \frac{1}{1}\right\} .
$$

The elements of the Farey sequence are called Farey fractions.

Fractions which are neighboring terms in any Farey sequence are known as a Farey pair. For example, $(0 / 1,1 / 1)$ and $(3 / 5,2 / 3)$ are two Farey pairs as their Farey fractions are neighbors in $F_{1}$ and $F_{5}$, respectively. For any Farey pair $a_{i} / b_{i}$ and $a_{j} / b_{j}$ it holds that $\left|a_{i} b_{j}-a_{j} b_{i}\right|=1$.

The mediant of a Farey pair, $a_{i} / b_{i}$ and $a_{j} / b_{j}$, is defined as $\left(a_{i}+a_{j}\right) /\left(b_{i}+b_{j}\right)$ and it is the only Farey fraction in between the Farey pair for the Farey sequence of order $b_{i}+b_{j}$. This allows to express any Farey fraction in between a Farey pair as a succession of mediant operations. 
Therefore, the numerator of the Farey fraction is a linear combination of the numerators of the Farey pair fractions (similarly for the denominators). This can be illustrated for any Farey fraction $h / k$ and for the Farey pair $(0 / 1,1 / 1)$ as

$$
\frac{h}{k}=\frac{(k-h) \cdot 0+h \cdot 1}{(k-h) \cdot 1+h \cdot 1} .
$$

The following fundamental properties of the gcd are recalled:

$$
\begin{aligned}
\operatorname{gcd}(x, 0) & =|x| \\
\operatorname{gcd}(m x, m y) & =m \operatorname{gcd}(x, y), \quad m>0 \\
\operatorname{gcd}(x+n y, y) & =\operatorname{gcd}(x, y) .
\end{aligned}
$$

\section{Results}

Theorem 2.1. Let $a_{i}$ and $b_{i}$ be positive integers such that $\operatorname{gcd}\left(a_{i}, b_{i}\right)=1$ for $i \in\{1,2,3\}$. Let $d_{i j}=\left|a_{i} b_{j}-a_{j} b_{i}\right|$, then

$$
\operatorname{gcd}\left(d_{32}, d_{31}\right)=\operatorname{gcd}\left(d_{32}, d_{21}\right)=\operatorname{gcd}\left(d_{31}, d_{21}\right)
$$

Proof. If $a_{i}>b_{i}$ for any $i \in\{1,2,3\}$ we can define new $\hat{b}_{i}$ as $\hat{b}_{i}=b_{i}+a_{i}$ for all $i \in\{1,2,3\}$ and $\hat{d}_{i j}=\left|a_{i} \hat{b}_{j}-a_{j} \hat{b}_{i}\right|$ so that $\hat{d}_{i j}=d_{i j}$ for all $i, j \in\{1,2,3\}$. Therefore it is sufficient to demonstrate Theorem 2.1 restricting to the case $a_{i} \leq b_{i}$ for all $i \in\{1,2,3\}$. In this form, the three fractions $a_{i} / b_{i}$ are Farey fractions. Furthermore, without loss of generality, we assume that the fractions are in the following order,

$$
\frac{a_{1}}{b_{1}} \leq \frac{a_{2}}{b_{2}} \leq \frac{a_{3}}{b_{3}} .
$$

The proof is divided in the following five cases:

1) $b_{1}=b_{2}=b_{3}$,

2) $b_{1}<b_{2}$ and $b_{1}<b_{3}$, which is symmetric to $b_{3}<b_{2}$ and $b_{3}<b_{1}$,

3) $b_{2}<b_{1}$ and $b_{2}<b_{3}$,

4) $b_{2}=b_{1}<b_{3}$, which is symmetric to $b_{2}=b_{3} \leq b_{1}$,

5) $b_{1}=b_{3}<b_{2}$.

For case 1) let $b$ be $b=b_{1}=b_{2}=b_{3}$ to express the three gcds of the theorem as:

$$
\begin{aligned}
& \operatorname{gcd}\left(d_{32}, d_{31}\right)=b \operatorname{gcd}\left(\left|a_{3}-a_{2}\right|,\left|a_{3}-a_{1}\right|\right), \\
& \operatorname{gcd}\left(d_{32}, d_{21}\right)=b \operatorname{gcd}\left(\left|a_{3}-a_{2}\right|,\left|a_{2}-a_{1}\right|\right), \\
& \operatorname{gcd}\left(d_{31}, d_{21}\right)=b \operatorname{gcd}\left(\left|a_{3}-a_{1}\right|,\left|a_{2}-a_{1}\right|\right) .
\end{aligned}
$$

These three quantities are equal as can be seen by using the property $\operatorname{gcd}(x, y)=\operatorname{gcd}(x, y \pm x)$. 
For case 2) $b_{1}<b_{2}$ and $b_{1}<b_{3}: a_{1} / b_{1}$ belongs to $F_{b_{1}}$ and there exists $a_{1}^{\prime} / b_{1}^{\prime}$ which forms a Farey pair with $a_{1} / b_{1}$ such that

$$
\frac{a_{1}}{b_{1}} \leq \frac{a_{2}}{b_{2}} \leq \frac{a_{3}}{b_{3}} \leq \frac{a_{1}^{\prime}}{b_{1}^{\prime}} .
$$

As discussed in Section 1 it is possible to express $a_{2} / b_{2}$ and $a_{3} / b_{3}$ by a succession of mediant operations starting from the Farey pair $a_{1} / b_{1}$ and $a_{1}^{\prime} / b_{1}^{\prime}$ as

$$
\frac{a_{2}}{b_{2}}=\frac{\left(k_{1}-h_{1}\right) a_{1}+h_{1} a_{1}^{\prime}}{\left(k_{1}-h_{1}\right) b_{1}+h_{1} b_{1}^{\prime}} \text { and } \frac{a_{3}}{b_{3}}=\frac{\left(k_{2}-h_{2}\right) a_{1}+h_{2} a_{1}^{\prime}}{\left(k_{2}-h_{2}\right) b_{1}+h_{2} b_{1}^{\prime}}
$$

with $h_{i} / k_{i}$ being Farey fractions. Computing $d_{i j}$ gives

$$
d_{32}=\left|h_{1} k_{2}-h_{2} k_{1}\right|, \quad d_{31}=h_{2}, \quad d_{21}=h_{1} .
$$

The equalities in the theorem are obtained following the gcd properties.

For case 3) $b_{2}<b_{1}$ and $b_{2}<b_{3}: a_{2} / b_{2}$ belongs to $F_{b_{2}}$ and there exist $a_{2}^{\prime} / b_{2}^{\prime}$ and $a_{2}^{\prime \prime} / b_{2}^{\prime \prime}$ which form two Farey pairs with $a_{2} / b_{2}$ such that

$$
\frac{a_{2}^{\prime}}{b_{2}^{\prime}} \leq \frac{a_{1}}{b_{1}} \leq \frac{a_{2}}{b_{2}} \leq \frac{a_{3}}{b_{3}} \leq \frac{a_{2}^{\prime \prime}}{b_{2}^{\prime \prime}}
$$

again applying a succession of mediant operations between $a_{2}^{\prime} / b_{2}^{\prime}$ or $a_{2}^{\prime \prime} / b_{2}^{\prime \prime}$ and $a_{2} / b_{2}$ yields

$$
\frac{a_{1}}{b_{1}}=\frac{\left(k_{1}-h_{1}\right) a_{2}^{\prime}+h_{1} a_{2}}{\left(k_{1}-h_{1}\right) b_{2}^{\prime}+h_{1} b_{2}} \text { and } \frac{a_{3}}{b_{3}}=\frac{\left(k_{2}-h_{2}\right) a_{2}+h_{2} a_{2}^{\prime \prime}}{\left(k_{2}-h_{2}\right) b_{2}+h_{2} b_{2}^{\prime \prime}}
$$

with $h_{i} / k_{i}$ being Farey fractions. Computing $d_{i j}$ gives

$$
\begin{gathered}
d_{32}=h_{2}, \quad d_{21}=k_{1}-h_{1}, \\
d_{31}=\left|\left(k_{1}-h_{1}\right)\left(k_{2}-h_{2}\right)+h_{1} h_{2}+h_{2}\left(k_{1}-h_{1}\right)\left(a_{2}^{\prime} b_{2}^{\prime \prime}-a_{2}^{\prime \prime} b_{2}^{\prime}\right)\right| .
\end{gathered}
$$

The equalities in the theorem are obtained following the gcd properties.

In case 4) let $b$ be $b=b_{2}=b_{1}<b_{3}$. The initial Farey fractions are transformed as $\frac{x}{y} \mapsto \frac{x}{y+x}$, resulting in

$$
\frac{a_{1}}{b+a_{1}}<\frac{a_{2}}{b+a_{2}}<\frac{a_{3}}{b_{3}+a_{3}} .
$$

This order preserving transformation within Farey fractions leaves unchanged the considered $d_{i j}$ and since $b+a_{1}<b+a_{2}$ and $b+a_{1}<b_{3}+a_{3}$ we are in case 2), for which the proof has already been given.

In case 5) let $b$ be $b=b_{1}=b_{3}<b_{2}$. Applying the same transformation as in case 4),

$$
\frac{a_{1}}{b+a_{1}}<\frac{a_{2}}{b_{2}+a_{2}}<\frac{a_{3}}{b+a_{3}}
$$

results in $b+a_{1}<b+a_{3}$ and $b+a_{1}<b_{2}+a_{2}$, which is case 2 ), for which the proof has already been given.

\section{References}

[1] Hardy, G. H., \& Wright, E. M. (1996). An Introduction to the Theory of Numbers, Fifth Edition, Oxford Science Publications. 